05

\title{
Магнитостатическая энергия доменных стенок в одноосных пленках конечных размеров
}

\author{
(С П.М. Ветошко, Ф.П. Ветошко, В.Г. Шавров, В.И. Щеглов \\ Институт радиотехники и электроники им. В.А. Котельникова РАН, \\ Москва, Россия \\ E-mail: pvetoshko@mail.ru
}

Поступила в Редакцию 10 октября 2018 г.

В окончательной редакции 16 апреля 2019 г.

Принята к публикации 26 апреля 2019 г.

\begin{abstract}
Приводится решение задачи определения магнитостатической энергии взаимодействия доменных стенок в одноосных магнетиках с однородным распределением намагниченности внутри доменов. Метод вычисления использует принцип эквивалентных токов, заключающийся в представлении намагниченности объекта с однородным распределением намагниченности эквивалентными токами, протекающими вдоль доменных границ и по поверхности. Получены аналитические выражения для взаимоиндукции двух прямоугольных проводников с произвольным соотношением сторон. Результаты могут быть полезны при определении равновесных конфигураций доменных структур в магнитных элементах спинтронных устройств, магнитных сенсоров и магнитной памяти.
\end{abstract}

Ключевые слова: магнитостатика, одноосная анизотропия, перемагничивание тонких пленок, доменная структура одноосных магнитных пленок.

DOI: 10.21883/FTT.2019.10.48247.278

\section{1. Введение}

Вычисление равновесной доменной конфигурации внутри магнитных элементов является сложной, трудоемкой задачей при проектировании и разработке спинтронных устройств, элементов магнитной памяти и магнитных сенсоров. Очевидно, что точное аналитическое выражение для магнитостатической энергии взаимодействия доменных стенок (ДС) может существенно упростить разработку элементов магнитных устройств, а в ряде случаев и подсказать новые инженерные решения и физические эффекты. Тем не менее, основная часть расчетов магнитостатической энергии ДС выполняется численными методами [1]. В первую очередь, это связано с отсутствием простых методик вычисления магнитостатической энергии взаимодействия ДС, а также существенным прогрессом в развитии методов микромагнитного моделирования, которые с успехом применяются для расчета распределения намагниченности в нано и микро размерных магнетиках [2].

Однако, в большом количестве прикладных задач возникает потребность расчета и анализа равновесных состояний магнетика с объемом порядка $1 \mathrm{~mm}^{3}$ и более. Так для магнитной пленки феррита-граната толщиной $10 \mu \mathrm{m}$ и размером порядка $10 \mathrm{~mm}$ минимально необходимый объем память компьютера составляет более $10^{12}$ байт [1]. Только в отдельных случаях возможно упрощение расчетной задачи с применением аналитического предсказания положения равновесия магнетика [3].

На сегодняшний день основными расчетными формулами для магнитостатики доменных структур являются выражения, полученные в известных работах Койя и
Энца [4], и Бобека [5] по магнитостатике уединенного полосового домена. Однако эти выражения получены в приближении бесконечной малости одного из геометрических размеров магнетика. В современных устройствах магнитной памяти, такие приближения недопустимы, поскольку все размеры магнитного элемента одного порядка.

В настоящей работе получены аналитические выражения для магнитостатической энергии взаимодействия ДС с учетом конечных размеров образца во всех трех измерениях. Для вычисления магнитостатической энергии был использован метод эквивалентных токов, использованный в [6] для определения областей устойчивости цилиндрического магнитного домена. В этом методе, энергия взаимодействия ДС представляется энергией взаимодействия поверхностных токов, протекающих по поверхности объема с однородной намагниченностью. Таким образом, вычисление магнитостатической энергии взаимодействия ДС сводится к вычислению взаимной индуктивности токов, ограничивающих области однородной намагниченности. Следует отметить серьезное упрощение для вычисления магнитостатической энергии в [6]. Поскольку намагниченность одноосных пленок является постоянной по направлению и величине, в выражении для магнитостатической энергии были опущены члены с намагниченностью магнетика, а учтены только те, что описывают энергию взаимодействия ДС. Таким образом, использованное приближение допустимо только при условии постоянства величины вектора М, т. е. его нельзя использовать, если предполагается взятие производных по величине и направлению намагниченности. Кроме того, аналитические выражения, использованные в [6], получены в приближении бесконечной 
малости одного из размеров структуры (в данном случае высоты доменной стенки по отношению к ее длине).

Аналитические выражения, полученные в данной работе, позволяют снять последнее ограничение, что особенно актуально для расчета структур с соизмеримыми размерами во всех трех направлениях, таких как элементы магнитной памяти, ячейки магнитооптических транспарантов и др. Несмотря на то, что данный метод расчета энергии ДС учитывает только ту часть магнитостатического потенциала, которая связана с взаимодействием ДС, в одноосных магнетиках такое приближение позволяет находить равновесные состояния конфигурации ДС например, определить область стабильного существования цилиндрического магнитного домена [6].

\section{2. Результаты и обсуждение}

Магнитостатическую энергию ДС одноосного магнетика можно представить как энергию контура с постоянным током $I=2 M_{s} h$, протекающим перпендикулярно намагниченности по доменным границам, и $I=M_{s} h-$ по границам элемента, где $h-$ толщина пленки, $M_{s}-$ намагниченность (рис. 1) [4].

Известно, что энергию системы токов можно представить, как

$$
\varepsilon=-\frac{1}{2} \sum_{i} \sum_{j} L_{i j} I_{i} I_{j},
$$

где $L_{i j}-$ взаимная индуктивность контуров с токами $I_{i}$ и $I_{j}[7]$.

Таким образом, расчет магнитостатической энергии ДС магнитного элемента с одноосной анизотропией сводится к расчету взаимных индуктивностей контуров, ограничивающих области однородной намагниченности. В случае прямоугольной геометрии элемента расчет сводится к определению взаимоиндукции между параллельными плоскими шинами, как показано на рис. 1.

В работах $[4,5]$ по магнитостатике уединенного полосового домена, длина доменной стенки (или эквивалентной тонкой шины) была принята много больше ширины и расстояния до соседней стенки или края образца, поэтому выражения для энергии доменной границы, полученные в $[4,5]$, не пригодны в случае, когда все три линейных размера магнетика соизмеримы.

Рассмотрим задачу об определении взаимоиндукции двух параллельных тонких шин при произвольном соотношении величин расстояния между шинами - $a$, длины $-b$ и ширины $-h$ (рис. 2).

Для вычисления коэффициента взаимоиндукции воспользуемся соотношением для взаимной индуктивности двух элементов объема $d V_{i}$ и $d V_{j}$ с плотностями протекающих внутри токов $J_{i}$ и $J_{j}[7]$ :

$$
L_{i j}=\frac{\mu_{0}}{4 \pi} \frac{1}{I_{i} I_{j}} \iint \frac{J_{i} J_{j}}{r_{i j}} d V_{i} d V_{j},
$$

где $I_{i}$ и $I_{j}-$ суммарные токи протекающие по элементам объемов интегрирования $d V_{i}$ и $d V_{j}$. Для токов,

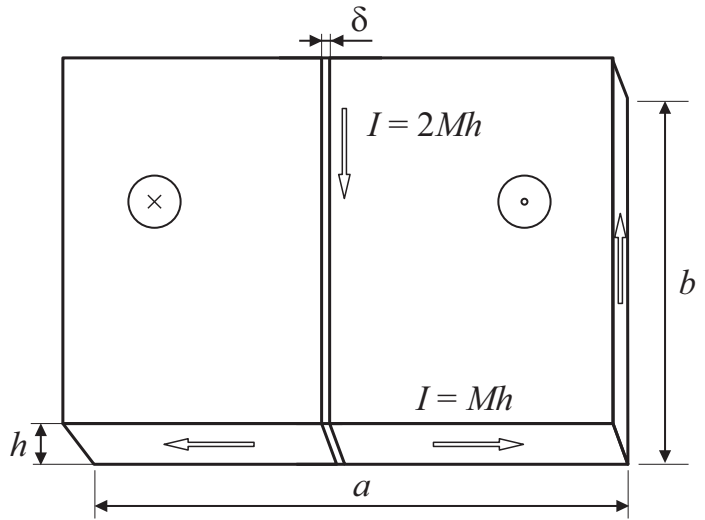

Рис. 1. Представление намагниченности с помощью эквивалентных поверхностных токов. Ток в доменной стенке в два раза больше тока по поверхности.

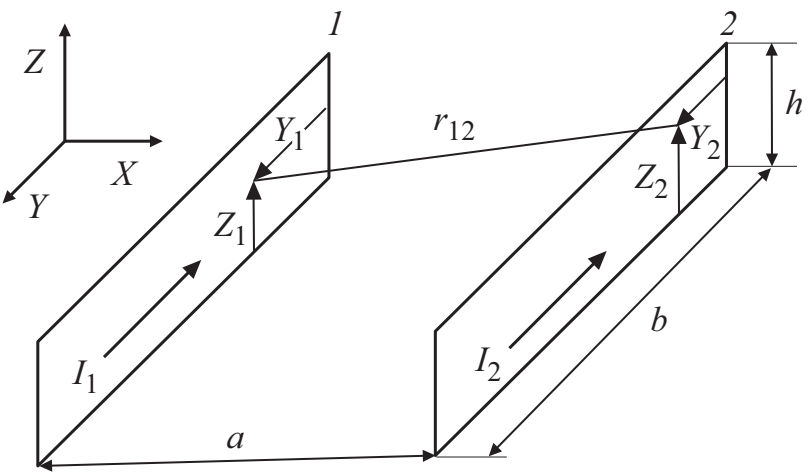

Рис. 2. Две параллельные тонкие шины с током. $I_{1,2}-$ направление тока в первой и второй шинах соответственно, расстояния между шинами $-a$, длина $-b$ и ширина $-h$.

протекающих по двум тонким проводам, эта формула переходит в формулу Неймана для двух линейных элементов тока [7]:

$$
L_{i j}=\frac{\mu_{0}}{4 \pi} \iint \frac{\cos \epsilon d S_{i} d S_{j}}{r_{i j}}
$$

где $\epsilon-$ угол между элементами тока, $r_{i j}-$ расстояние между элементами тока.

В случае двух плоскостей $i=1, j=2$ (рис. 2), формула (2) приходит к виду

$$
L_{12}=\frac{\mu_{0}}{4 \pi b^{2}} \iiint \int \frac{1}{r_{12}} d Z_{1} d Z_{2} d Y_{1} d Y_{2}
$$

где $r_{12}=\sqrt{a^{2}+\left(Z_{1}-Z_{2}\right)^{2}+\left(Y_{1}-Y_{2}\right)^{2}}-$ расстояние между элементами интегрирования $d Z_{1} d Y_{1}$ и $d Z_{2} d Y_{2}$, как показано на рис. 2.

Подробности вычисления интеграла (4) приведены в Приложении 1. 
Окончательное выражение для $L_{i j}$, применимое при любых соотношениях $a, b$ и $h$, имеет вид

$$
\begin{aligned}
& L_{i j}(b, a, h)=\frac{\mu_{0}}{12 \pi h^{2}}\left[2\left(P_{h}^{3}+P_{a}^{3}-P^{3}-a^{3}\right)\right. \\
& -6 a^{2}\left(P_{h}+P_{a}-P-a\right)-12 a b h \tan ^{-1}\left(\frac{b h}{a P}\right) \\
& +3 h\left(b^{2}-a^{2}\right) \ln \frac{P+h}{P-h}+3 a^{2} h \ln \frac{P_{b}+h}{P_{b}-h} \\
& \left.+6 a^{2} b \ln \frac{\left(b+P_{a}\right) P_{h}}{(b+P) a}+6 h^{2} b \ln \frac{b+P}{P_{h}}\right]
\end{aligned}
$$

где $P=\sqrt{a^{2}+h^{2}+b^{2}}, P_{a}=\sqrt{a^{2}+b^{2}}, P_{h}=\sqrt{a^{2}+h^{2}}$.

В Приложениях 2,3 даны некоторые асимптотические выражения для формулы (5). Показано, что в приближении бесконечно длинных доменных стенок выражение для магнитостатического поля на доменной границе, полученное путем предельного перехода из выражения (5) $\lim _{b \rightarrow \infty} \frac{d L_{12}}{d(a)}$, совпадает с полученным ранее в работе [4].

Поскольку в выражении (5) нет ограничений на соотношение величин $b, a$ и $h$, то построив поверхность магнетика из прямоугольных граней, можно определить магнитостатическую энергию в структурах с произвольным соотношением размеров сторон.

Следующим затруднением для определения взаимной индуктивности параллельных проводов является параллельное смещение граней относительно друг друга.

Как было показано в [9], существует общее правило для определения взаимной индуктивности $L_{12}$ параллельных проводов $m$ и $n$ со смещением $k$ (рис. 3,a), согласно которому, определение взаимной индуктивности $L_{12}$ может быть сведено к определению взаимных индуктивностей нескольких пар проводов, а именно:

$$
2 L_{12}=L_{\alpha}-L_{\beta}-L_{\gamma}+L_{\delta}
$$

где $L_{\alpha}, L_{\beta}, L_{\gamma}, L_{\delta}$ - находятся по формуле для $L_{12}$ (П1.2) при значениях $b$ (рис. 3) $b=\alpha, \beta, \gamma$ и $\delta$ соответственно. При этом: $\alpha=m+n+k ; \beta=m+n ; \gamma=n+k ; \delta=k$. Также предполагается, что токи вдоль отрезков $m$ и $n$ протекают в одном направлении.

Поскольку формула (5) была получена из (П1.2) путем интегрирования по $h$, а равенство (6) выполняется для любых двух пар проводников в интеграле (П1.3), то правило (6) также применимо для индуктивностей, получаемых по формуле (5). На рис. $3, b$ и $c$ приведены два частных случая взаимных положений отрезков $m$ и $n$, и соответствующие выражения для определения взаимной индуктивности.

В качестве примера определим магнитостатический потенциал прямоугольного элемента с одноосной анизотропией, разделенного прямой, вертикальной доменной стенкой (рис. 4). Будем полагать, что размеры элемента не превосходят размера равновесного домена и доменная стенка 6 на рис. 4 устойчива.

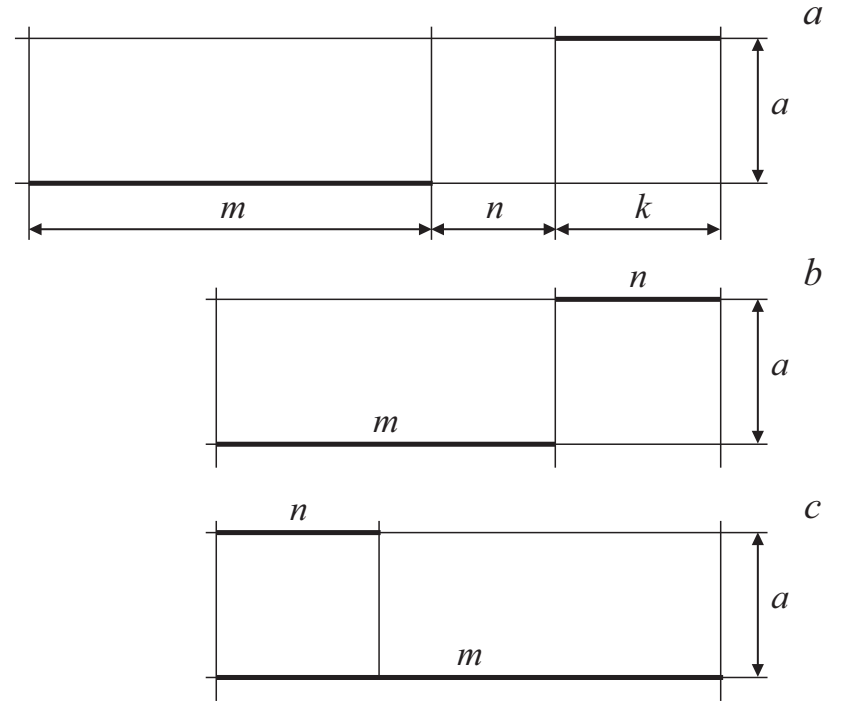

Рис. 3. Различные конфигурации взаимного расположения проводов; $a$ - параллельные провода в общем случае; величины отрезков $m$ и $k$ - длины проводов, $n-$ сдвиг вдоль провода, $a-$ расстояние между проводами; $b-$ частный случай взаимного расположения отрезков $m$ и $n$, провода $m$ и $n$ расположены напротив друг друга; $c-$ провода $m$ и $n$ расположены параллельно со сдвигом.

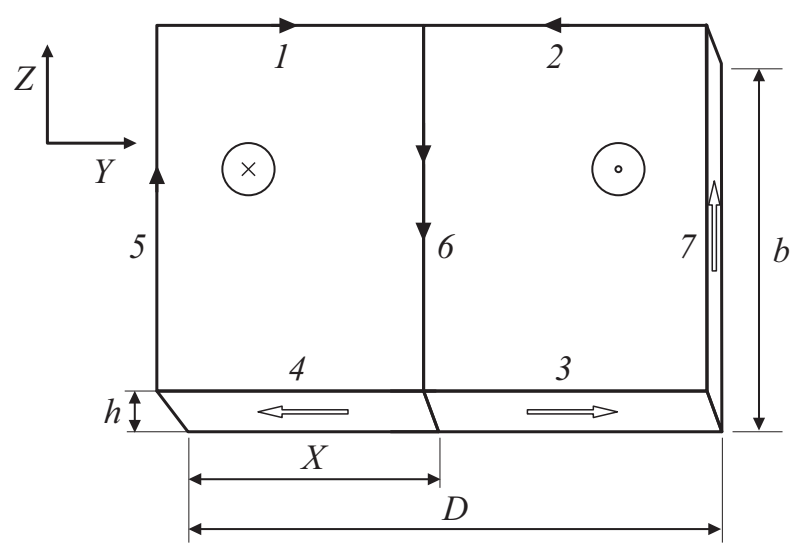

Рис. 4. Прямоугольный элемент с одноосной анизотропией и одиночной доменной границей. Границе 6 приписывается удвоенное значение эквивалентного тока.

Запишем магнитостатическую энергию как сумму энергий элементов с током $I=M_{s} h$ (для отрезка 6 ток равен $2 I)$ с учетом направления тока

$$
\begin{aligned}
-\frac{2 \varepsilon}{\left(M_{s} h\right)^{2}} & =L_{11}-L_{12}+L_{13}-L_{14}-L_{21}+L_{22} \\
& -L_{23}+L_{24}+L_{31}-L_{32}+L_{33}-L_{34}-L_{41} \\
& +L_{42}-L_{43}+L_{44}+L_{55}-2 L_{56}+L_{57} \\
& -2 L_{65}+4 L_{66}-2 L_{67}+L_{75}-2 L_{76}+L_{77} .
\end{aligned}
$$


Далее с учетом симметрии элемента

$$
\begin{gathered}
L_{11}=L_{44}, \quad L_{22}=L_{33}, L_{12}=L_{43}, \\
L_{13}=L_{24}, \quad L_{55}=L_{66}=L_{77},
\end{gathered}
$$

а также с учетом взаимности $L_{i j}=L_{j i}$, выражение (7) примет вид

$$
\begin{aligned}
-\frac{2 \varepsilon}{\left(M_{s} h\right)^{2}}= & 2 L_{11}+2 L_{22}+4 L_{13}-4 L_{12}-2 L_{14} \\
& -2 L_{23}+6 L_{55}-4 L_{56}-4 L_{67}+2 L_{57} .
\end{aligned}
$$

Выражение (6) может быть разделено на две части по направлению протекания тока, $\varepsilon_{I Z}$ - энергия взаимодействия токов коллинеарных направлению $Z$ и $E_{I Y}$ для токов перпендикулярного направления $Y$. Такая запись удобна в прямоугольной геометрии элемента, поскольку взаимная индуктивность перпендикулярных элементов равна нулю (3)

$$
\begin{gathered}
\varepsilon_{I Z}=-\frac{1}{2}\left(M_{s} h\right)^{2}\left[6 L_{55}-4 L_{56}-4 L_{67}+2 L_{57}\right] \\
\varepsilon_{I Y}=-\frac{1}{2}\left(M_{s} h\right)^{2}\left[2 L_{11}+2 L_{22}+4 L_{13}-4 L_{12}-2 L_{14}-2 L_{23}\right]
\end{gathered}
$$

Согласно выражению (5) будем обозначать индуктивность между двумя элементами как $L(b, a, h)-$ где $b-$ длина в направлении протекания тока, $a-$ расстояние между проводниками, $h$ - ширина проводника. В случае если индексы проводников совпадают (самоиндукция проводника), переменную а будем приравнивать нулю. Выражение для самоиндукции проводника получено предельным переходом при $a \rightarrow 0$, и приведено в Приложении 2 (П2.3).

С помощью правил (6) можно выписать значения индуктивностей в (9):

$$
\begin{gathered}
L_{11}=L(x, 0, h), \\
L_{22}=L(D-x, 0, h), \\
L_{12}=\frac{1}{2}[L(D, 0, h)-L(x, 0, h)-L(D-x, 0, h)], \\
L_{13}=\frac{1}{2}[L(D, b, h)-L(x, b, h)-L(D-x, b, h)], \\
L_{14}=L(x, b, h), \\
L_{23}=L(D-x, b, h), \\
L_{55}=L(b, 0, h), \\
L_{56}=L(b, x, h), \\
L_{57}=L(b, D, h), \\
L_{67}=L(b, D-x, h) .
\end{gathered}
$$

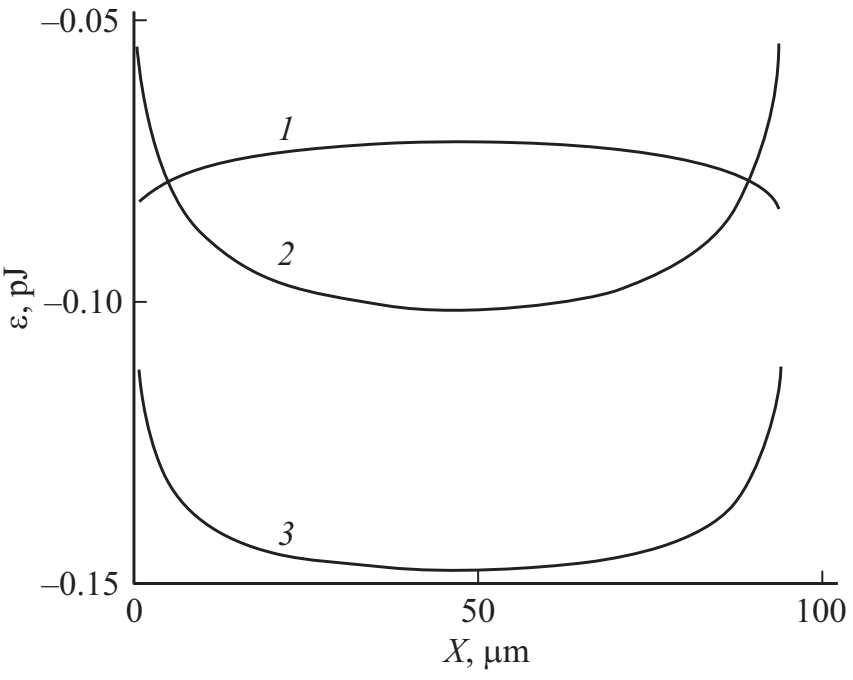

Рис. 5. Магнитостатический потенциал в зависимости от смещения доменной границы по координате $Y$ (3), отдельно показаны энергия взаимодействия токов вдоль направления $Y-$ $\varepsilon_{I Y}(1)$ и вдоль направления $Z-\varepsilon_{I Z}(2)$. Энергии (1) и (2) могут быть использованы для взаимной компенсации с целью получения состояния безразличного равновесия доменной границы.

Окончательное выражение для магнитостатического потенциала по смещению доменной стенки $x$

$$
\begin{aligned}
\varepsilon= & -\frac{1}{2}\left(M_{s} h\right)^{2}[4 L(x, 0, h)+4 L(D-x, 0, h) \\
& -2 L(D, 0, h)-4 L(x, b, h)-4 L(D-x, b, h) \\
& +2 L(D, b, h)+6 L(b, 0, h)-4 L(b, x, h) \\
& -4 L(b, D-x, h)+2 L(b, D, h)]
\end{aligned}
$$

На рис. 5 изображены зависимости магнитостатической энергии элемента от смещения доменной стенки вдоль координаты $Y$. Энергия $\varepsilon_{I Z}$, обусловленная протеканием тока вдоль оси $Z$, и энергия $\varepsilon_{I Y}$, возникающая при взаимодействии токов вдоль оси $Y$, имеют противоположный характер зависимости от координаты $Y$, что указывает на возможность взаимной компенсации при соответствующем выборе геометрии элемента. Такая геометрия в виде прямоугольника с элементами моста была экспериментально реализована в работе [10].

\section{3. Заключение}

В работе предложена методика расчета энергии взаимодействия ДС в одноосном магнетике, состоящая из следующих последовательных процедур:

1) представление поверхности магнетика перпендикулярными плоскостями;

2) представление намагниченности магнетика поверхностным током, протекающим перпендикулярно намагниченности вдоль этих плоскостей; 
3) подсчет взаимоиндукций отдельных плоскостей;

4) представление энергии взаимодействия ДС как энергии системы токов протекающим вдоль ДС и по границам магнетика.

Приведенная методика расчета позволяет ускорить процедуру вычисления магнитостатической энергии, что необходимо для создания и оптимизации формы магнитных элементов с заданными параметрами доменной структуры.

\section{Приложение 1. Вычисление взаимной индуктивности двух параллельных тонких шин с током}

Рассмотрим две параллельные плоскости размерами $h$ и $b$ отнесенными друг от друга на расстояние $a$ (рис. 2). Тогда расстояние между точками на плоскостях с координатами $\left(Z_{1}, Y_{1}\right)$ и $\left(Z_{2}, Y_{2}\right)$ может быть записано как,

$$
r_{12}=\sqrt{a^{2}+\left(Z_{1}-Z_{2}\right)^{2}+\left(Y_{1}-Y_{2}\right)^{2}},
$$

а вычисление взаимоиндукции между шинами $L_{12}$ сводится к четырехкратному интегралу типа

$$
\iiint \int \frac{1}{\sqrt{a^{2}+\left(Z_{1}-Z_{2}\right)^{2}+\left(Y_{1}-Y_{2}\right)^{2}}} d Z_{1} d Z_{2} d Y_{1} d Y_{2} .
$$

Пределы интегрирования $[0, b]-$ по оси $Y$, и $[0, h]-$ по оси $Z$.

Двойное интегрирование по $Y$ приводит к хорошо известной формуле для взаимной индуктивности двух линейных токов:

$$
L_{12}=\frac{\mu_{0}}{2 \pi}\left[b \log \frac{b \sqrt{b^{2}+a^{2}}}{a}-\sqrt{b^{2}+a^{2}}+a\right] .
$$

Чтобы получить выражение для взаимоиндукции двух тонких шин, необходимо два раза проинтегрировать (П1.2) по ширине шины $h$, при этом в выражении (3) необходимо заменить а на $\sqrt{\left(a^{2}+(x-y)^{2}\right.}$

$$
\begin{aligned}
L_{12}= & \frac{\mu_{0}}{2 \pi h^{2}} \int_{0}^{h} \int\left[b \log \frac{b \sqrt{b^{2}+a^{2}+(x-y)^{2}}}{a}\right. \\
& \left.-\sqrt{b^{2}+a^{2}+(x-y)^{2}}+\sqrt{a^{2}+(x-y)^{2}}\right] d x d y .
\end{aligned}
$$

Для упрощения преобразований при вычислении (П1.3) учтем симметрию функции $(x-y)^{2}$ относительно линии $x=y$, это позволяет в первом интегрировании по $x$ сделать подстановку с заменой пределов интегрирования: $(x-y)^{2} \rightarrow x^{2}$, $[0, h] \rightarrow[-y, h-y]$.
В этом случае второе интегрирование по у сведется к взятию интеграла вида:

$$
\begin{aligned}
& L_{12}=\frac{\mu_{0}}{2 \pi h^{2}} \int_{0}^{h}\left[y \sqrt{a^{2}+y^{2}}-y \sqrt{a^{2}+y^{2}+b^{2}}\right. \\
& +h \sqrt{h^{2}+a^{2}+y^{2}-2 h y}-y \sqrt{h^{2}+a^{2}+y^{2}-2 h y} \\
& -h \sqrt{h^{2}+a^{2}+y^{2}+b^{2}-2 h y} \\
& +y \sqrt{h^{2}+a^{2}+y^{2}+b^{2}-2 h y} \\
& -2 a b \arctan \left[\frac{b y}{a \sqrt{a^{2}+y^{2}+b^{2}}}\right] \\
& -2 a b \arctan \left[\frac{b(h-y)}{a \sqrt{h^{2}+a^{2}+y^{2}+b^{2}-2 h y}}\right] \\
& -a^{2} \log \left[-y+\sqrt{a^{2}+y^{2}}\right]+2 b y \log \left[\frac{b+\sqrt{a^{2}+y^{2}+b^{2}}}{\sqrt{a^{2}+y^{2}}}\right] \\
& +a^{2} \log \left[-y+\sqrt{a^{2}+y^{2}+b^{2}}\right] \\
& -b^{2} \log \left[-y+\sqrt{a^{2}+h^{2}+b^{2}}\right] \\
& +a^{2} \log \left[h-y+\sqrt{h^{2}+a^{2}+y^{2}-2 h y}\right] \\
& +2 h b \log \left[\frac{b+\sqrt{h^{2}+a^{2}+y^{2}+b^{2}-2 h y}}{\sqrt{h^{2}+a^{2}+y^{2}-2 h y}}\right] \\
& -2 b y \log \left[\frac{b \sqrt{h^{2}+a^{2}+y^{2}+b^{2}-2 h y}}{\sqrt{h^{2}+a^{2}+y^{2}-2 h y}}\right] \\
& -a^{2} \log \left[h-y+\sqrt{h^{2}+a^{2}+y^{2}+b^{2}-2 h y}\right] \\
& \left.+b^{2} \log \left[h-y+\sqrt{h+a^{2}+y^{2}+b^{2}-2 h y}\right]\right] d y .
\end{aligned}
$$

После интегрирования по $y$ :

$$
\begin{aligned}
& L_{12}=\frac{\mu_{0}}{12 \pi h^{2}}\left(4 a^{2} \sqrt{h^{2}+a^{2}+b^{2}}\right. \\
& -2 h^{2} \sqrt{h^{2}+a^{2}+b^{2}}-2 b^{2} \sqrt{h^{2}+a^{2}+b^{2}} \\
& -12 a b h \tan ^{-1}\left(\frac{b h}{a \sqrt{h^{2}+a^{2}+b^{2}}}\right) \\
& +6 a^{2} b \log \frac{\sqrt{h^{2}+a^{2}}\left(\sqrt{a^{2}+b^{2}}-b\right)\left(\sqrt{h^{2}+a^{2}+b^{2}}-b\right)}{a} \\
& -3 h b^{2} \log \left(\frac{-2 h \sqrt{h^{2}+a^{2}+b^{2}}+2 h^{2}+a^{2}+b^{2}}{a^{2}+b^{2}}\right) \\
& +6 h a^{2} \log \frac{\left(\sqrt{h^{2}+a^{2}}-h\right)\left(\sqrt{h^{2}+a^{2}+b^{2}}+h\right)}{a\left(\sqrt{h^{2}+a^{2}+b^{2}}-h\right)} \\
& +6 h^{2} b \log \frac{\sqrt{h^{2}+a^{2}+b^{2}}+b}{\sqrt{h^{2}+a^{2}}} \\
& -4 a^{2} \sqrt{h^{2}+a^{2}}+2 h^{2} \sqrt{h^{2}+a^{2}}+4 a^{3} \\
& \left.-4 a^{2} \sqrt{a^{2}+b^{2}}+2 b^{2} \sqrt{a^{2}+b^{2}}\right) .
\end{aligned}
$$


Подставляя $P=\sqrt{a^{2}+h^{2}+b^{2}}, \quad P_{a}=\sqrt{a^{2}+b^{2}}, \quad P_{h}=$ $=\sqrt{a^{2}+h^{2}}$ в (П1.5) окончательно получаем:

$$
\begin{aligned}
L_{12} & =\frac{\mu_{0}}{12 \pi h^{2}}\left[2\left(P_{h}^{3}+P_{a}^{3}-P^{3}-a^{3}\right)\right. \\
& -6 a^{2}\left(P_{h}+P_{a}-P-a\right)-12 a b h \tan ^{-1}\left(\frac{b h}{a P}\right) \\
& +3 h\left(b^{2}-a^{2}\right) \ln \frac{P+h}{P-h}+3 a^{2} h \ln \frac{P_{b}+h}{P_{b}-h} \\
& \left.+6 a^{2} b \ln \frac{\left(b+P_{a}\right) P_{h}}{(b+P) a}+6 h^{2} b \ln \frac{b+P}{P_{h}}\right] .
\end{aligned}
$$

\section{Приложение 2. Вычисление собственной индуктивности}

Собственная индуктивность тонкой шины может быть получена путем предельного перехода при $a \rightarrow 0$

$$
L_{i i}=\lim _{a \rightarrow 0} L_{i j}
$$

Подставляя сюда выражение для $L_{i j}($ П1.5), имеем

$$
\begin{aligned}
& L_{i i}=\frac{\mu_{0}}{12 \pi h^{2}}\left(4 a^{2} \sqrt{h^{2}+a^{2}+b^{2}}\right. \\
& -2 h^{2} \sqrt{h^{2}+a^{2}+b^{2}}-2 b^{2} \sqrt{h^{2}+a^{2}+b^{2}} \\
& -12 a b h \tan ^{-1}\left(\frac{b h}{a \sqrt{h^{2}+a^{2}+b^{2}}}\right) \\
& +6 a^{2} b \log \frac{\sqrt{h^{2}+a^{2}}\left(\sqrt{a^{2}+b^{2}}-b\right)\left(\sqrt{h^{2}+a^{2}+b^{2}}-b\right)}{a} \\
& -3 h b^{2} \log \left(\frac{-2 h \sqrt{h^{2}+a^{2}+b^{2}}+2 h^{2}+a^{2}+b^{2}}{a^{2}+b^{2}}\right) \\
& +6 h a^{2} \log \frac{\left(\sqrt{h^{2}+a^{2}}-h\right)\left(\sqrt{h^{2}+a^{2}+b^{2}}+h\right)}{a\left(\sqrt{h^{2}+a^{2}+b^{2}}-h\right)} \\
& +6 h^{2} b \log \frac{\sqrt{h^{2}+a^{2}+b^{2}}+b}{\sqrt{h^{2}+a^{2}}}-4 a^{2} \sqrt{h^{2}+a^{2}} \\
& \left.+2 h^{2} \sqrt{h^{2}+a^{2}}+4 a^{3}-4 a^{2} \sqrt{a^{2}+b^{2}}+2 b^{2} \sqrt{a^{2}+b^{2}}\right) .
\end{aligned}
$$

После преобразований:

$$
\begin{aligned}
L_{i i} & =\frac{\mu_{0}}{12 \pi h^{2}}\left(2 h^{3}+2 b^{3}-2\left(h^{2}+b^{2}\right)^{3 / 2}\right. \\
& \left.+6 h^{2} b \log \frac{b+\sqrt{h^{2}+b^{2}}}{h}-6 h b^{2} \log \frac{\sqrt{h^{2}+b^{2}}-h}{b}\right) .
\end{aligned}
$$

Чтобы сравнить с имеющимися выражениями для индуктивности тонкой шины, разложим полученное выра- жение в ряд по $h$

$$
L_{i i}=\frac{\mu_{0}}{2 \pi}\left(\log \frac{2 b}{h}+\frac{1}{2}+\frac{h}{3 b}-\frac{h^{2}}{24 b^{2}}+\frac{h^{4}}{480 b^{4}}+O\left(\frac{h^{6}}{b^{6}}\right)\right) .
$$

Наиболее полное выражение для индуктивности тонкой шины, полученное в [8], соответствует первым трем членам разложения (П2.4).

\section{Приложение 3. Вычисление величины эффективного магнитного поля, действующего на доменную границу}

Для расчета величины эффективного магнитного поля, действующего на доменную границу необходимы выражения для производных индуктивности по линейному смещению:

$$
\begin{aligned}
& \frac{\partial L_{i i}}{\partial b}=\frac{\mu_{0}}{2 \pi h^{2}}\left(b^{2}-b \sqrt{h^{2}+b^{2}}\right. \\
& \left.+b^{2} \log \frac{b+\sqrt{h^{2}+b^{2}}}{h}-2 b h \log \frac{\sqrt{h^{2}+b^{2}}-h}{b}\right), \\
& \frac{\partial L_{i i}}{\partial b}=-\frac{\mu_{0}}{4 \pi h^{2}}\left(4 a h \tan ^{-1}\left(\frac{b h}{a \sqrt{h^{2}+a^{2}+b^{2}}}\right)\right. \\
& -2 b\left(\sqrt{a^{2}+b^{2}}-\sqrt{h^{2}+a^{2}+b^{2}}\right) \\
& -4 h b \log \frac{\sqrt{h^{2}+a^{2}+b^{2}}+h}{\sqrt{a^{2}+b^{2}}} \\
& -2 a^{2} \log \frac{\left(b+\sqrt{a^{2}+b^{2}}\right)\left(\sqrt{h^{2}+a^{2}+b^{2}}-b\right)}{a \sqrt{a^{2}+h^{2}}} \\
& \left.-2 h^{2} \log \frac{b+\sqrt{h^{2}+a^{2}+b^{2}}}{a^{2}+h^{2}}\right), \\
& \frac{\partial L_{i i}}{\partial a}=-\frac{\mu_{0}}{\pi h^{2}}\left(a h \tan ^{-1}\left(\frac{b h}{a \sqrt{h^{2}+a^{2}+b^{2}}}\right)\right. \\
& +a\left(\sqrt{a^{2}+b^{2}}-\sqrt{h^{2}+a^{2}+b^{2}}+\sqrt{a^{2}+h^{2}}-a\right) \\
& +\frac{1}{2} h a \log \frac{\left(\sqrt{h^{2}+a^{2}+b^{2}}+h\right)\left(\sqrt{a^{2}+h^{2}}-h\right)}{a \sqrt{a^{2}+b^{2}}} \\
& \left.+a b \log \frac{\sqrt{a^{2}+h^{2}}\left(\sqrt{a^{2}+b^{2}}-b\right)}{a\left(\sqrt{h^{2}+a^{2}+b^{2}}-b\right)}\right) .
\end{aligned}
$$

Заметим, что выражение (П3.3) определяет поле на доменной стенке в полосовом домене. Если приравнять пондеромоторные силы, связанные с изменением взаимоиндукции между токовыми шинами высотой $h$ и длиной $b$ к силе в эффективном магнитном поле $B[6]$ :

$$
2 h b M B=-I^{2} \frac{\partial L_{i j}}{\partial a} .
$$


Подставляя $I=2 M h-$ эффективный ток вдоль доменной границы, получаем:

$$
\frac{B}{\mu_{0} M}=\frac{2 h}{b} \frac{\partial L_{i j}}{\partial a}
$$

если выражение в правой части (П3.5) устремить к бесконечности по $b$ - длине доменной стенке, то в пределе получаем:

$$
\lim _{b \rightarrow \infty} \frac{2 h}{b} \frac{\partial L_{i j}}{\partial a}=\frac{2}{\pi}\left(\tan ^{-1}\left(\frac{h}{a}\right)-\frac{a}{2 h} \log \left(1+\frac{h^{2}}{a^{2}}\right)\right)
$$

- известное выражение [5], полученное при рассмотрении магнитостатики полосового домена.

\section{Благодарности}

Авторы признательны А.И. Морозову за обсуждение работы и многочисленные полезные замечания.

\section{Финансирование}

Работа выполнена в рамках государственного задания.

\section{Конфликт интересов}

Авторы заявляют, что у них нет конфликта интересов.

\section{Список литературы}

[1] M.J. Donahue. IEEE Transact. Magn. 45, 3923 (2009).

[2] F. Bruckner, C. Abert, G. Wautischer, C. Huber, C. Vogler, M. Hinze, D. Suess. Sci. Rep. 7, 40816 (2017).

[3] Petr Vetoshko, Vladimir Skidanov, A. Stempkovskiy. Sensor Lett. 11, 59 (2013).

[4] C. Kooy, U. Enz. Phyl. Res. Rep. 15, 7 (1960).

[5] A.H. Bobeck. Bell Syst. Techn. J. 46, 1901 (1967).

[6] T.H. O’Dell. Magnetic bubbles. The Macmillan Press Ltd, N.Y. (1974). P. 27-56.

[7] А.Н. Матвеев. Электродинамика. Высш. шк., М. (1980). $383 \mathrm{c}$.

[8] E.B. Rosa. Bull. Bureau Stand. 4, 2, 301 (1908).

[9] П.Л. Калантаров, Л.А. Цейтлин. Расчет индуктивностей. Энергоатомиздат, Л. (1986). 488 с.

[10] V.A. Skidanov, P.M. Vetoshko, A.L. Stempkovsky. J. Phys.: Conf. Ser. 266, 012125 (2011).

Редактор Т.Н. Василевская 